\title{
Measurement of the specific heat of a fractional quantum Hall system
}

\author{
F. Schulze-Wischeler, ${ }^{1, *}$ U. Zeitler, ${ }^{2}$ C. v. Zobeltitz, ${ }^{3}$ F. Hohls, ${ }^{1}$ D. Reuter, ${ }^{4}$ A. D. Wieck, ${ }^{4}$ H. Frahm, ${ }^{3}$ and R. J. Haug ${ }^{1}$ \\ ${ }^{1}$ Institut für Festkörperphysik, Leibniz Universität Hannover, Appelstraße 2, 30167 Hannover, Germany \\ ${ }^{2}$ High Field Magnet Laboratory, Institute for Molecules and Materials, Radboud University Nijmegen, Toernooiveld 7 , \\ 6525 ED Nijmegen, The Netherlands \\ ${ }^{3}$ Institut für Theoretische Physik, Leibniz, Universität Hannover, Appelstraße 2, 30167 Hannover, Germany \\ ${ }^{4}$ Lehrstuhl für Angewandte Festkörperphysik, Ruhr-Universität Bochum, Universitätsstraße 150, 44780 Bochum, Germany
}

(Received 7 June 2007; revised manuscript received 21 August 2007; published 25 October 2007)

\begin{abstract}
Using a time-resolved phonon absorption technique, we have measured the relative specific heat of a two-dimensional electron system in the fractional quantum Hall effect regime. For filling factors $\nu=5 / 3,4 / 3$, $2 / 3,3 / 5,4 / 7,2 / 5$, and $1 / 3$ the specific heat displays a strong exponential temperature dependence in agreement with excitations across a quasiparticle gap. At filling factor $\nu=1 / 2$ we were able to measure the relative specific heat of a composite fermion system. The observed nearly linear temperature dependence on temperature down to $T=0.14 \mathrm{~K}$ agrees well with early predictions for a Fermi liquid of composite fermions.
\end{abstract}

DOI: $10.1103 /$ PhysRevB.76.153311

PACS number(s): 73.43.-f, 72.10.Di, 73.20.Mf

In the fractional quantum Hall (FQH) effect the Coulomb interaction induces the formation of new quasiparticle states characterized by a fractional charge and a finite excitation gap. ${ }^{1-4}$ A nice description of the FQH effect is given by the composite fermion (CF) theory ${ }^{5}$ where the FQH effect is regarded as an integer quantum Hall effect of new quasiparticles. In particular, at a filling factor $\nu=1 / 2$ these composite fermions can be described as a quasi-free Fermi sea in an effective zero-magnetic field. ${ }^{6}$

One of the experimental challenges lies in accessing the ground state properties of a two-dimensional electron system (2DES) by means of thermodynamic quantities such as magnetization, ${ }^{7-9}$ thermal conductivity, ${ }^{10,11}$ thermopower, ${ }^{12,13}$ or specific heat. ${ }^{14,15}$ It is, however, far from being straightforward to measure the specific heat $C$ of a 2DES directly. In general $C$ is strongly dominated by the contribution of the surrounding substrate, and, in order to obtain a reasonable signal from the $2 \mathrm{DES}$, multilayer structures have to be used. ${ }^{14,15}$

An alternative method lies in the use of time-resolved phonon-spectroscopy experiments. ${ }^{16,17,26}$ Here, a defined amount of energy is dissipated inside the 2DES in a short period of time (typically a few nanoseconds). Measuring the temperature of the 2DES long before it reaches equilibrium with the substrate then allows us to determine its specific heat directly. ${ }^{18}$

In this Brief Report we will show how we can use phonon-absorption experiments to deduce the temperature dependence of $C$ of a $2 \mathrm{DES}$ in the FQH regime. Results at fractional filling factors suggest the excitation across a quasiparticle gap in quantitative agreement with theoretical predictions. ${ }^{19}$ At filling factor $\nu=1 / 2$ we essentially find a linear temperature dependence predicted for a Fermi liquid of composite fermions. ${ }^{6}$ Slight deviations from linearity will be tracked down to spin splitting in the CF system.

The sample used in this experiment is a 2DES embedded in a modulation-doped AlGaAs/GaAs heterostructure. Using its persistent photoconductivity the electron concentration $n_{e}$ inside the 2DES can be tuned be means of an infrared photodiode. The sample is mounted on the cold finger of a dilution refrigerator and inserted into a $13 \mathrm{~T}$ superconducting magnet. In order to heat up the 2DES on short time scales of a few nanoseconds, it is bombarded with ballistic-phonon pulses emitted from a heater on the back side of the $2 \mathrm{~mm}$ thick GaAs substrate; a more detailed description of this phonon absortion technique can be found elsewhere. ${ }^{17,18,20}$ The temperature change inside the 2DES is measured by converting its time-dependent resistance $R$ to a temperature $T$ using the equilibrium calibration for $R(T)$. In order to maximize the signal to noise ratio, the $2 \mathrm{DES}$ is patterned into a long meander, see Ref. 20 for more details.

Figure 1(a) shows a typical phonon signal for a 2DES at filling factor $\nu=1 / 2$ at different equilibrium temperatures $T_{0}$ between 75 and $500 \mathrm{mK}$. At $t=0$ a $10 \mathrm{~ns}$ phonon pulse is emitted. This pulse is characterized by a nonequilibrium phonon temperature $T_{P}=1.9 \mathrm{~K}$. After $0.6 \mu \mathrm{s}$ the phonon pulse reaches the 2DES which leads to an increase of its temperature to a maximum peak height $T_{1}$. The magnitude of the phonon signal, $\Delta T=T_{1}-T_{0}$, decreases with increased $T_{0}$. This fact is emphasized in Fig. 1(b) where we have plotted $\Delta T$ as a function of $T_{0}$. As we will quantify furthermore the
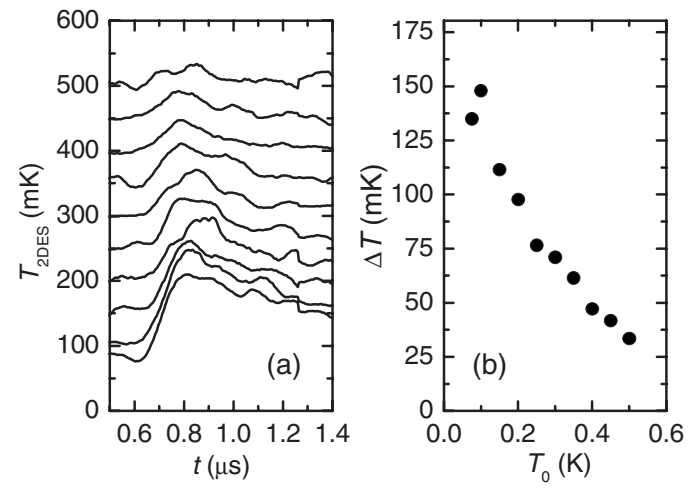

FIG. 1. Phonon signal data at $\nu=1 / 2\left(n_{e}=1.03 \times 10^{15} \mathrm{~m}^{-2}\right)$. (a) Phonon signal in $\mathrm{mK}$ versus time for same phonon temperature $T_{P}=1.9 \mathrm{~K}$ and pulse length $\tau=10 \mathrm{~ns}$. From bottom to top the equilibrium temperature of the 2DES is increased from 75 to $500 \mathrm{mK}$. (b) Total temperature change $\Delta T$ of the 2DES due to ballisticphonon absorption as a function of $T_{0}$ as used in (a). 
monotonic decrease of $\Delta T$ is directly related to a monotonic increase of the 2DES specific heat at $\nu=1 / 2$.

In order to extract the 2DES specific heat from this phonon-absorption experiments we introduce a simple model: The temperature change $d T$ of a 2DES due to phonon absorption can be written as

$$
C(T) d T=r\left(T, T_{P}\right) P_{P} d t,
$$

where $C(T)$ is the 2DES's specific heat, and $r\left(T, T_{P}\right)$ is an absorption coefficient depending on the nonequilibrium phonon temperature $T_{P}$ and 2DES temperature $T . P_{P}$ is total power inside one phonon pulse. Since phonon emission cooling the 2DES back to its equilibrium temperature takes place at much longer time scales (typically in microns) it can be neglected on the $10 \mathrm{~ns}$ time scale for phonon absorption. Integrating this general equation over the phonon-pulse length $\tau$ leads to

$$
\int_{T_{0}}^{T 1} C(T) d T=r_{0} P_{P} \tau
$$

In the experiment the phonon temperature $T_{P}$ is kept constant, and the absorption coefficient $r\left(T, T_{P}\right)$ only depends on the temperature $T$. For low enough temperatures $T \ll T_{P}$ the 2DES will be essentially in its (temperature independent) ground state and $r$ can be regarded as constant. This is indeed the case for our experiments which justifies the use of a roughly constant absorption rate $r_{0}$ for all temperatures used.

Using Eq. (2) we can then determine the (relative) specific heat of the 2DES, $C(T) / r_{0}$, from a set of experiments as shown in Fig. 1. Although $r_{0}$ is a priori unknown this procedure already allows a direct access to the temperature dependence of the specific heat of a 2DES.

Results for the specific heat of $\mathrm{FQH}$ filling factors $\nu=5 / 3,4 / 3,2 / 3,3 / 5,4 / 7,2 / 5$, and $1 / 3$ are shown in Fig. 2 ; the corresponding electron concentrations $n_{e}$ are marked in the figure. Please note that the measurements were taken at different $n_{e}$ because filling factors $5 / 3,4 / 3,3 / 5$, and $4 / 7$ showed up only at highest electron concentration (due to higher electron mobility of the illuminated sample), whereas $\nu=1 / 3$, was only accessible for lower electron concentrations in our magnet, $2 / 3$ and $2 / 5$, and shows data at a medium electron concentration. All the curves are taken for different electron concentrations and show the same characteristic form: for low temperatures $T$ the relative specific heat $C(T) / r_{0}$ is flat and finite, and increases exponentially for higher temperatures.

The theoretically predicted specific heat of such a system is $C \propto(1 / T)^{2} \exp (-\Delta / T) .{ }^{19}$ Here $\Delta$ is the energy gap from the occupied ground state to the following unoccupied Landau level of composite fermions. The solid lines shown in Fig. 2 show a comparison of this theory with our experimental data. We have added a small empirical constant caused by a finite (thermodynamic) density of states inside the excitation gap. ${ }^{18}$ The energy gaps $\Delta$ deduced from this procedure (see Fig. 2) are comparable to gaps determined from temperature dependent transport experiments, ${ }^{20,21}$ but show a large uncertainty due to fits far away from the temperatures corresponding to the gab energies, especially at $\nu=1 / 3$.

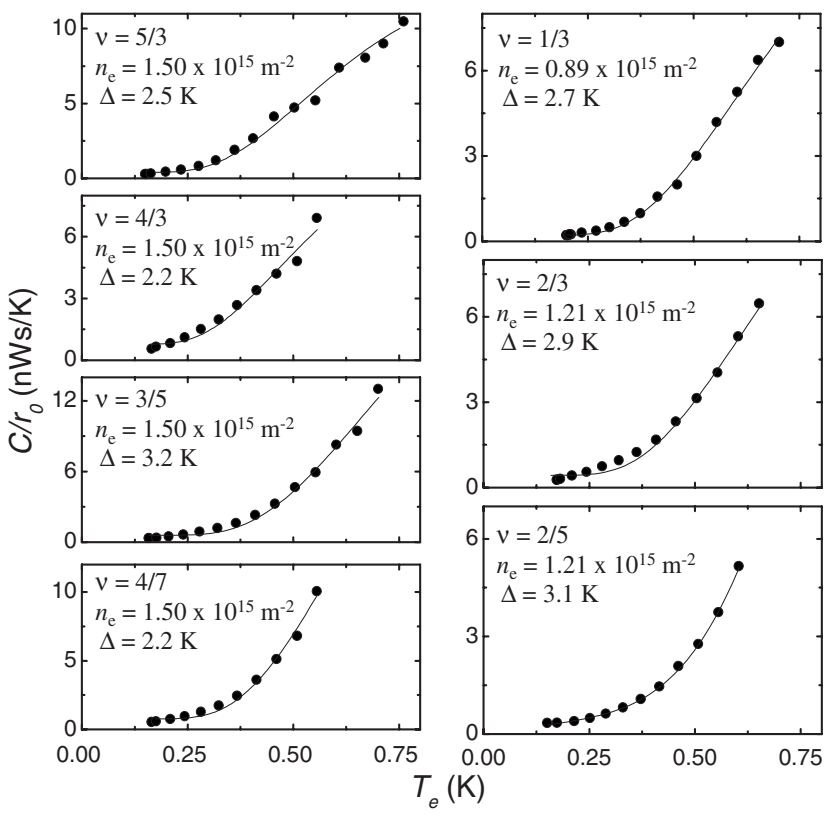

FIG. 2. Relative specific heat $C(T) / r_{0}$ for the filling factors $\nu=5 / 3,4 / 3,2 / 3,3 / 5,4 / 7,2 / 5$, and $1 / 3$ measured with a phononabsorption experiment. The unknown absorption coefficient $r_{0}, 0<r_{0}<1$, is fixed for every single graph. The lines are fits with the theory (see text).

The temperature dependence of the specific heat at $\nu=1 / 2$ is distinctively different from the fractional filling factors shown above. Figure 3 shows our results for relative specific heat $C(T) / r_{0}$ at $\nu=1 / 2$ for eight different electron densities ranging from $n_{e}=0.89 \times 10^{15} \mathrm{~m}^{-2}$ to $n_{e}$ $=1.50 \times 10^{15} \mathrm{~m}^{-2}$. Note that all measurements are done with the same standard phonon pulse ( $\tau=10 \mathrm{~ns}, T_{P}=1.9 \mathrm{~K}$ ). Since the phonon signal at $\nu=1 / 2$ is extremely weak we had to

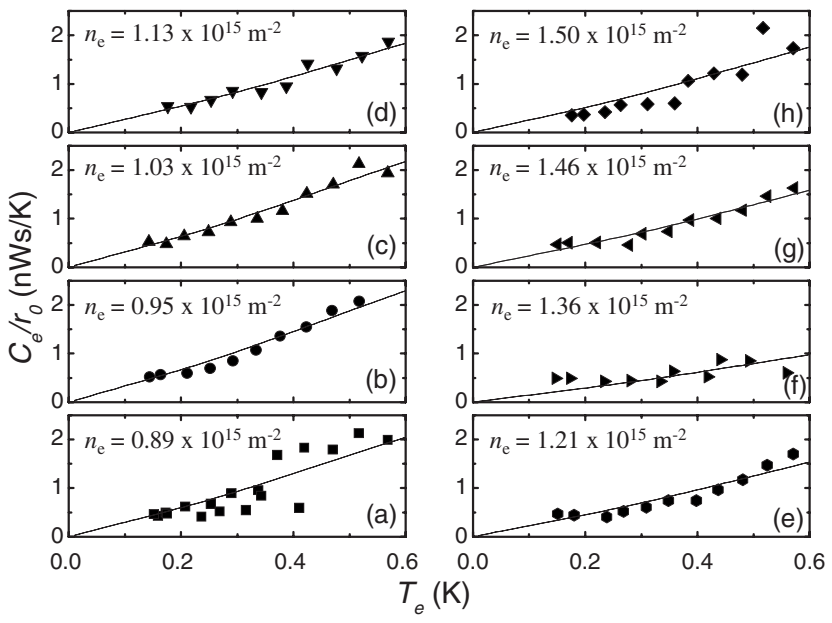

FIG. 3. Relative specific heat $C(T) / r_{0}$ at filling factor $\nu=1 / 2$ for eight different electron densities from $n_{e}=0.89 \times 10^{15} \mathrm{~m}^{-2}$ (a) up to $n_{e}=1.50 \times 10^{15} \mathrm{~m}^{-2}(\mathrm{~h})$ measured in a phonon absorption experiment. The lines are fits with the theoretical expected linear behavior through the origin (Ref. 6) plus a term $\left(\Delta_{\text {spin }}^{2} / T\right) e^{-\Delta_{\text {spin }} / k_{B} T}$ taking the influence of a second constant density of states into account. The error of the data points is given by their distribution. 
average over up to $1.5 \times 10^{6}$ individual traces in order to obtain reliable data. The reason for the weakness of the signal (and therefore the large amount of scatter in the data) is that the resistance nearly not changes as a function of temperature at $\nu=1 / 2$. For higher temperatures the resistance change goes nearly to zero and for this physical reason the specific heat at $T>0.6 \mathrm{~K}$ cannot be measured with phononabsorption experiments here at all. The measurements strongly suggest a linear temperature dependence of the specific heat for small $T$.

This agrees with the $\mathrm{CF}$ picture of the $\mathrm{FQH}$ effect (FQHE) in mean-field approximation (i.e., neglecting fluctuations of the fictitious gauge field introduced in the transformation to $\mathrm{CFs}$ ) as a consequence of quasiparticle and quasihole close excitations close to the Fermi surface. ${ }^{6}$ Our experimental observation therefore forms another strong confirmation of the CF picture of the FQHE.

The dependence of the relative specific heat $C(T) / r_{0}$ on electron concentration $n_{e}$ is governed by two effects. On the one hand, the composite-fermion mass $m_{C F}$ increases proportional to $\sqrt{n_{e}} \cdot 6,22$ On the other hand, the coupling of TA phonons to a $2 \mathrm{DES}$ is piezoelectric and therefore proportional to the electron density $n_{e},{ }^{17,23}$ i.e., $r_{0} \propto n_{e}$. These two competing processes lead to a concentration dependence of the relative specific heat $C(T) / r_{0} \propto 1 / \sqrt{n_{e}}$ consistent with our experimental data.

Beyond the leading linear temperature dependence the analysis of our data gives a small positive contribution to $C$. This is different from the CF picture for a single half-filled Landau level where gauge fluctuations leading to logarithmic corrections to the entropy are expected to reduce the specific heat. ${ }^{6}$

To explain this behavior we propose a description taking into account the spin of the CF: ${ }^{24,25}$ at the magnetic field $B_{1 / 2}$ corresponding to filling factor $\nu=1 / 2$ the ground state of the system is completely spin polarized. The second Landau level for the electrons with opposite spin is separated from the Fermi energy $E_{F}$ by a finite gap $\Delta_{s p i n}=g^{*} \mu_{B} B_{1 / 2}-E_{F}$ (see Fig. 4). Here $g^{*}$ is the effective Landé factor of the CFs.

Within this picture, the total energy $E_{t o t}$ of the system at a finite temperature $T$ is

$$
E_{t o t}=\int_{0}^{\infty} \frac{D E}{1+e^{\beta[E-\mu(T)]}} d E+\int_{E_{F}+\Delta_{s p i n}}^{\infty} \frac{D E}{1+e^{\beta[E-\mu(T)]}} d E .
$$

The second term represents the contribution of the second spin state to the total energy with the constant density of

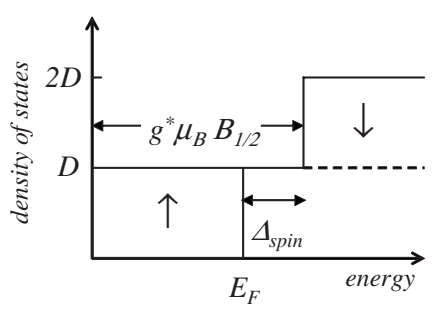

FIG. 4. Model for the density of states at $\nu=1 / 2$ including spin. At $T=0$ the system is spin polarized and filled with spin-up CF up to the Fermi energy $E_{F}$. The second (spin-down) band is shifted by the Zeeman splitting $g^{*} \mu_{B} B_{1 / 2}$, the lowest lying separated from $E_{F}$ by an energy gap $\Delta_{\text {spin }}$.

states $D$ of the CF system. Computing these integrals the leading terms of the specific heat are found to be

$$
C=-\frac{\partial E_{t o t}}{\partial T} \approx \frac{\pi^{2}}{3} D k_{B} T+D \frac{\Delta_{\text {spin }}^{2}}{k_{B} T} e^{-\Delta_{s p i n} / k_{B} T} .
$$

Please note that the first linear term is the known result from Halperin, Lee, and Read $C=\frac{\pi}{6} m_{C F} k_{B}^{2} T^{6}$ by inserting the density of states $D=N / E_{F}$ and the Fermi energy of free fermions (CFs in our case) $E_{F}=\frac{\hbar^{2}}{2 m_{C F}}(4 \pi N)$.

We have fitted our data in Fig. 3 with Eq. (4) and find indeed a good agrement. The energy gap $\Delta_{\text {spin }}$ determined by this procedure is $\Delta_{\text {spin }}(\nu=1 / 2)=1.7 \pm 0.3 \mathrm{~K}$, independent on the electron concentration. This means implicitly that the g-factor decreases when the electron concentration is increased.

In conclusion, we have measured the temperature dependence of the relative specific heat of a two-dimensional electron system in the fractional quantum Hall system at various filling factors. The results depend strongly on the filling factor and can be classified into two groups: Measurements at filling factors $\nu=5 / 3,4 / 3,2 / 3,3 / 5,4 / 7,2 / 5$, and $1 / 3$, where the sample is in a state with an energy gap $\Delta$, show a clear exponential behavior. In contrast, the measured relative specific heat at filling factor $\nu=1 / 2$, corresponding to a Fermi sea of composite fermions, strongly suggests a linear temperature dependence; small deviations from this linearity could be explained with the influence of a second spin state at $\nu=1 / 2$.

We acknowledge financial support by DFG priority program "quantum Hall systems" and discussions with W. Apel (PTB Braunschweig), W. Weller (ITP Leipzig), and K. v. Klitzing (MPI-Fkf Stuttgart).

\footnotetext{
*Present address: Laboratorium für Nano- und Quantenengineering, Leibniz Universität Hannover, Schneiderberg 32, 30167 Hannover, Germany.

${ }^{1}$ D. C. Tsui, H. L. Stormer, and A. C. Gossard, Phys. Rev. Lett. 48, 1559 (1982).

${ }^{2}$ R. B. Laughlin, Phys. Rev. Lett. 50, 1395 (1983).
}

${ }^{3}$ Composite Fermions, edited by O. Heinonen (World Scientific, Singapore, 1998).

${ }^{4}$ Perspectives in Quantum Hall Effects, edited by S. Das Sarma and A. Pinczuk (Wiley, New York, 1997).

${ }^{5}$ J. K. Jain, Phys. Rev. Lett. 63, 199 (1989).

${ }^{6}$ B. I. Halperin, P. A. Lee, and N. Read, Phys. Rev. B 47, 7312 
(1993).

${ }^{7}$ J. P. Eisenstein, H. L. Stormer, V. Narayanamurti, A. Y. Cho, A. C. Gossard, and C. W. Tu, Phys. Rev. Lett. 55, 875 (1985).

${ }^{8}$ S. A. J. Wiegers, M. Specht, L. P. Lévy, M. Y. Simmons, D. A. Ritchie, A. Cavanna, B. Etienne, G. Martinez, and P. Wyder, Phys. Rev. Lett. 79, 3238 (1997).

${ }^{9}$ I. Meinel, T. Hengstmann, D. Grundler, D. Heitmann, W. Wegscheider, and M. Bichler, Phys. Rev. Lett. 82, 819 (1999).

${ }^{10}$ J. P. Eisenstein, A. C. Gossard, and V. Narayanamurti, Phys. Rev. Lett. 59, 1341 (1987).

${ }^{11}$ V. Bayot, M. B. Santos, and M. Shayegan, Phys. Rev. B 46, 7240 (1992).

${ }^{12}$ U. Zeitler, J. C. Maan, P. Wyder, R. Fletcher, C. T. Foxon, and J. J. Harris, Phys. Rev. B 47, 16008 (1993).

${ }^{13}$ B. Tieke, U. Zeitler, R. Fletcher, S. A. J. Wiegers, A. K. Geim, J. C. Maan, and M. Henini, Phys. Rev. Lett. 76, 3630 (1996); B. Tieke, R. Fletcher, U. Zeitler, M. Henini, and J. C. Maan, Phys. Rev. B 58, 2017 (1998).

${ }^{14}$ E. Gornik, R. Lassnig, G. Strasser, H. L. Stormer, A. C. Gossard, and W. Wiegmann, Phys. Rev. Lett. 54, 1820 (1985).

${ }^{15}$ E. Grivei, S. Melinte, V. Bayot, H. C. Manoharan, and M. Shayegan, Phys. Rev. B 68, 193404 (2003).

${ }^{16}$ C. J. Mellor, R. H. Eyles, J. E. Digby, A. J. Kent, K. A. Benedict,
L. J. Challis, M. Henini, C. T. Foxon, and J. J. Harris, Phys. Rev. Lett. 74, 2339 (1995).

${ }^{17}$ A. J. Kent, R. E. Strickland, K. R. Strickland, and M. Henini, Phys. Rev. B 54, 2019 (1996).

${ }^{18}$ U. Zeitler, A. M. Devitt, J. E. Digby, C. J. Mellor, A. J. Kent, K. A. Benedict, and T. Cheng, Phys. Rev. Lett. 82, 5333 (1999).

${ }^{19}$ T. Chakraborty and P. Pietiläinen, Phys. Rev. B 55, R1954 (1997).

${ }^{20}$ F. Schulze-Wischeler, F. Hohls, U. Zeitler, D. Reuter, A. D. Wieck, and R. J. Haug, Phys. Rev. Lett. 93, 026801 (2004).

${ }^{21}$ The energy gaps as measured from the specific heat are thermodynamic gaps susceptible to the disorder in the system. A method using phonon-absorption experiments always yields larger absolute gaps.

${ }^{22}$ J. K. Jain and R. K. Kamilla, Phys. Rev. B 55, R4895 (1997).

${ }^{23}$ K. A. Benedict, R. K. Hills, and C. J. Mellor, Phys. Rev. B 60, 10984 (1999).

${ }^{24}$ B. Kramer, E. Mariani, N. Magnoli, M. Merlo, F. Napoli, and M. Sassetti, Phys. Status Solidi B 234, 221 (2002).

${ }^{25}$ E. Mariani, N. Magnoli, F. Napoli, M. Sassetti, and B. Kramer, Phys. Rev. B 66, 241303(R) (2002).

${ }^{26}$ U. Zeitler, A. M. Devitt, J. E. Digby, C. J. Mellor, A. J. Kent, K. A. Benedict, and T. Cheng, Physica B 249, 49 (1998). 